\title{
ESTUDO COMPARATIVO ENTRE OS TESTES ADOTADOS PELO PNCEBT PARA O DIAGNÓSTICO SOROLÓGICO DA BRUCELOSE EM BOVINOS*
}

\section{R.B. Meirelles-Bartoli; L.A. Mathias}

Universidade Estadual Paulista, Faculdade de Ciências Agrárias e Veterinárias, Departamento de Medicina Veterinária Preventiva e Reprodução Animal, Via de Acesso Prof. Paulo Donato Castellane, s/nº, CEP 14884900, Jaboticabal, SP, Brasil. E-mail: raphaellabrasil@hotmail.com

\section{RESUMO}

\begin{abstract}
Avaliaram-se comparativamente as provas do antígeno acidificado tamponado (AAT), a combinação das provas de soroaglutinação lenta e 2-mercaptoetanol (2-ME) e a reação de fixação de complemento (RFC), provas preconizadas pelo Programa Nacional de Controle e Erradicação de Brucelose Tuberculose (PNCEBT). Para tanto, foram alisadas 1.061 amostras de soros bovinos. Os dados foram analisados pelo indicador kappa, adotando-se como ponto de corte o título 25 no 2-ME e 4 na RFC. Constatou-se sensibilidade relativa de 99,6\%, 98,8\% e 91,1\%, respectivamente, para o AAT, a 2-ME e a RFC, e especificidade relativa de $83,9 \%, 96,2 \%$ e 100,0\%. A comparação entre os testes adotados pelo programa apontou concordância boa entre o teste de triagem (AAT) e os testes confirmatórios (kappa: $2-\mathrm{ME}=0,80$; e $\mathrm{RFC}=0,73$ ) e concordância ótima quando os testes confirmatórios foram comparados entre si $(\mathrm{kappa}=0,86)$. No entanto foram encontrados soros com título elevado em um dos testes confirmatórios e resultado negativo no outro, o que reforça a ideia de queo diagnóstico sorológico da brucelose é mais confiável quando obtido por meio dos resultados de vários testes.
\end{abstract}

PALAVRAS-CHAVE: Brucelose bovina, diagnóstico sorológico, concordância, sensibilidade diagnóstica, especificidade diagnóstica.

\section{ABSTRACT}

COMPARATIVESTUDY OF SEROLOGICAL TESTS FOR BOVINE BRUCELLOSIS DIAGNOSIS ADOPTED BY THE BRAZILIAN PROGRAM FOR ANIMAL BRUCELLOSIS AND TUBERCULOSIS CONTROL AND ERADICATION.Serum samples from 1,061 bovine were analyzed by serological diagnostic techniques adopted by the Brazilian Program for Animal Brucellosis and Tuberculosis Control and Eradication in order to compare, as a screening test, the rose Bengal plate test (RBPT), and as confirmatory tests, the 2-mercaptoethanol plus standard tube agglutination test (2-ME), and the complement fixation test (CFT). Relative sensitivity of $99.6 \%, 98.8 \%$ and $91.1 \%$ were observed, respectively, for RBPT, 2-ME and CFT, and relative specificity of $83.9 \%, 96.2 \%$ and $100.0 \%$. The agreement between tests was analyzed by the kappa statistic $(2-\mathrm{ME}$ cut-off $=$ titer 25 , and CFT cutoff $=$ titer 4$)$. RBPT showed a substantial agreement (kappa: $2-\mathrm{ME}=0.80$; and $\mathrm{RFC}=0.73$ ) with the confirmatory tests, and excellent agreement between the confirmatory tests (kappa $=0.86$ ) was observed. However sera with a negative result in one of the confirmatory tests and a high titer in the other were observed, reinforcing that the serological diagnosis of bovine brucellosis is more reliable when based on the results of several tests.

KEY WORDS: Bovine brucellosis, serodiagnosis, agreement, sensitivity, specificity.

\section{INTRODUÇÃO}

A brucelose ainda é um dos principais problemas sanitários do rebanho bovino brasileiro. Em 2001, foi instituído pelo Ministério da Agricultura, Pecuária e Abastecimento (MAPA) o Programa Nacional de Controle e Erradicação da Brucelose e Tuberculose
Animal (PNCEBT), com o objetivo de diminuir o impacto negativo dessas zoonoses na saúde humana e animal, além de promover a competitividade da pecuária nacional (BRASIL, 2001).

O PNCEBT prevê que o diagnóstico da brucelose bovina e bubalina deve ser feito usando a prova do antígenoacidificado tamponado(AAT) como teste de

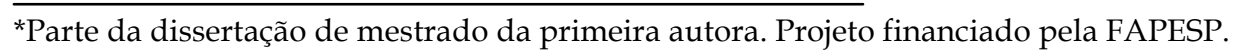


triagem, e os animais com resultado positivo nesse teste podem ser classificados como infectados ou então podem ser submetidos a um teste confirmatório, situação para a qual existem duas opções: a combinação das provas de soroaglutinação lenta e do 2mercaptoetanol (2-ME) ou então a reação de fixação de complemento (RFC) (BRASIL, 2001).

OAAT foi escolhido como teste de triagem por sua sensibilidade, rapidez e simplicidade de execução, porém, animais vacinados com a amostra B19 podem apresentar reações falso-positivas nesse teste (HUBER; Nicoletti, 1986; Nicolett; T ANYA, 1993; Abimerhi etal., 1998; Megid et al., 2000; Fosgate et al., 2002). Assim, provas confirmatórias de maior especificidade devem ser realizadas, para evitar o sacrifício de animais não infectados, porém, geralmente elas são mais caras e laboriosas (NIELSEN, 2002).

Apesar de o 2-ME ser eficiente para bovinos (Nicoletti, 1969; Mariño et al., 1991) e bubalinos (Shandu; Joshi, 1993; Bercovich, 1998; Fosgate et al., 2002), NIELSEN(2002) indicou a possibilidade de ocorrência de resultado falso-negativo nesse teste, em decorrência da presença de pontes dissulfeto também nas moléculas de IgG, e BERCovich (1998) alegou não ser uma prova adequada para a identificação de animais infectados recentemente.

A RFC é o teste de referência recomendado pela Organização Mundial de Saúde Animal (OIE) para o trânsito internacional de animais (DAJERet al., 1999) e tem sido utilizada com sucesso em muitos países nos programas de controle e erradicação (NIELSEN, 2002). Estudos realizados em bovinos infectados, natural ou experimentalmente, indicaram que a RFC apresenta boa correlação com o isolamento do agente etiológico (HAYES; ChAPPEL, 1982; Sutherland et al., 1982; CORNER et al., 1983), fato que embasou sua adoção como teste de referência para a avaliação de outros testes sorológicos (MATHIAS; PINTO, 1983; Mathias etal.,1995). Além da alta especificidade, a RFC sofre menor interferência dos anticorpos vacinais do que as provas do AAT e 2-ME (Alton et al., 1975).

Ambos os testes confirmatórios aceitos pelo PNCEBT possuem suas vantagens e desvantagens, e existem vários trabalhos comparando os resultados dos dois testes em bovinos (NICOLETTI, 1969; CORRÊADE SÁ, 1989; MARIÑo et al., 1991; MYlreA; Fraser, 1976; PAulin et al., 2002; Kuroda et al., 2004) e em bubalinos (PINToet al., 2005). A literatura também registra diversos artigos comparando resultados do teste de triagem com os testes confirmatórios em bovinos (GUARINO et al. 2001; KURODA et al., 2004; DAJER et al., 1999) e em bubalinos (PAulin, 2006; Molnár et al., 2002; PINTO et al., 2005), com resultados nem sempre apontando na mesma direção.

Diante disso, o trabalho em pauta se propôs a estudar o desempenho das provas oficiais do PNCEBT
(AAT, 2-ME e RFC) no diagnóstico sorológico da brucelose bovina.

\section{MATERIAL E MÉTODOS}

\section{Amostras}

Foram analisados soros sanguíneos de bovinos adultos, de diversas raças, ambos os sexos e de diferentes propriedades, encaminhados ao Laboratório de Diagnóstico de Brucelose do Departamento de Medicina Veterinária Preventiva e Reprodução Animal da Faculdade de Ciências Agrárias e Veterinárias (FCAV) da Unesp, Campus de Jaboticabal, SP, e também soros bovinos colhidos em frigoríficos do Estado de São Paulo, totalizando 1.061 amostras.

\section{Técnicas laboratoriais}

Os testes do AAT, SAL e 2-ME foram realizados conforme as técnicas recomendadas no Manual Técnico do Programa Nacional de Controle e Erradicação da Brucelose e Tuberculose (BRASIL, 2006). No teste do AAT, o antígeno empregadoé preparado com Brucella abortus amostra 1119/3, corado com rosa de Bengala, na concentração de 8,0\% de volume celular, $\mathrm{pH} 3,63$. Nos testes de SAL e 2-ME, foi utilizado antígeno de célula total (antígeno para soroaglutinação lenta), preparado com B.abortus amostra 1119/3, na concentração final de $0,045 \%$ de volume celular. A interpretação da combinação dos resultados do teste do 2-ME e do teste de SAL foi obtida segundo estabelece o PNCEBT; devido ao fato de não se conhecer a condição vacinal dos animais avaliados, adotou-se o quadro correspondente a animais não vacinados (BRASIL, 2001).

Na execução da reação de fixação de complemento, foi empregada a microtécnica com incubação a $37^{\circ}$ $C$ nas duas fases da reação, recomendada por AlTON et al. (1988). Foi utilizado antígeno produzido $\operatorname{com} B$. abortus amostra 1119/3, o mesmo usado no teste de soroaglutinação lenta em tubos. Como complemento foi utilizado soro de cobaia, titulado conforme a técnica descrita por Alton et al. (1988). Foi empregado sistema hemolítico formado por hemácias de carneiro sensibilizadas com hemolisina (anticorpo de coelho contra hemácia de ovino) titulada conforme a recomendação de Alton et al. (1988). Para a classificação dos resultados em positivos e negativos adotou-se a diluição 1:4 como ponto de corte.

\section{Análise dos dados}

Para a comparação entre os resultados das diversas provas foi usado o coeficiente Kappa, o qual foi 
interpretado de acordo com os critérios adotados por Pereira (2000). Para o cálculo da sensibilidade e da especificidade diagnóstica relativas de cada teste adotado pelo programa, a condição verdadeira foi estabelecida pela combinação dos resultados dos outros testes, conforme recomendam MARTiN et al. (1987), ou seja, amostras com resultado negativo nos outros dois testes foram consideradas de animais não infectados, amostras com resultado positivo nos outros dois testes foram consideradas de animais infectados e amostras com resultado discrepante entre os dois testes que serviram de parâmetro para determinar a condição verdadeira foram desconsideradas.

\section{RESULTADOS}

Os resultados obtidos na prova do AAT e na combinação das provas SAL+2-ME, conforme prevê a legislação brasileira (negativos, inconclusivos e positivos), podem ser observados na Tabela 1. Das 644 amostras positivas no AAT, 79 (12,3\%) foram negativas na combinaçãoSAL+2-ME, 37 (5,7\%) foram inconclusivas e $528(82,0 \%)$ tiveram o resultado positivo confirmado. Quanto às 417 amostras negativas no AAT, 376 (90,2\%) também foram negativas na combinação SAL+2-ME, 24 (5,8\%) foram inconclusivas, mas $17(4,1 \%)$ apresentaram resultado positivo. Desconsiderando as 61 amostras com resultado inconclusivo na combinação SAL+2-ME, observa-se uma concordância boa, pois o valor de kappa foi igual a 0,80 , e uma proporção de $90,4 \%$ de resultados concordantes.

Tabela 1 - Resultados do teste do antígeno acidificado tamponado (AAT) comparados com a interpretação da combinação dos resultados dos testes de soroaglutinação lenta (SAL) e 2-mercaptoetanol (2-ME) para diagnóstico sorológico de brucelose em bovinos.

\begin{tabular}{lcccc}
\hline AAT & \multicolumn{3}{c}{ SAL + 2-ME } & Total \\
\cline { 2 - 4 } & Positivo & Inconclusivo & Negativo & \\
\hline Positivo & 528 & 37 & 79 & 644 \\
Negativo & 17 & 24 & 376 & 417 \\
\hline Total & 545 & 61 & 455 & 1.061 \\
\hline
\end{tabular}

Das 644 amostras com resultado positivo no AAT, $494(76,7 \%)$ apresentaram resultado positivo também na RFC, ao passo que as outras 150 (23,3\%) apresentaram resultado negativo. Pôde-se observar também que, das 417 amostras com resultado negativo no AAT, $2(0,5 \%)$ apresentaram resultado positivo na
RFC, e as outras 415 (99,5\%) expressaram resultado negativo nessa prova (Tabela 2). A comparação entre esses dois testes resultou em uma concordância boa, com $85,7 \%$ de resultados concordantes e um indicador kappa igual a 0,73.

Tabela 2 - Resultados do teste do antígeno acidificado tamponado (AAT) em comparação com os títulos obtidos na reação de fixação de complemento (RFC) para diagnóstico sorológico de brucelose em bovinos.

\begin{tabular}{lrcr}
\hline AAT & \multicolumn{2}{c}{ RFC } & Total \\
\cline { 2 - 3 } & Positivo & Negativo & \\
\hline Positivo & 494 & 150 & 644 \\
Negativo & 2 & 415 & 417 \\
\hline Total & 496 & 565 & 1.061 \\
\hline
\end{tabular}

Quando comparados os resultados da RFC com os da combinação dos testes SAL+2-ME (Tabela 3), observa-se que, dos 455 soros com resultado negativo na combinação, $448(98,5 \%)$ apresentaram resultado negativo na RFC e 7 (1,5\%) apresentaram título a partir de 4, devendo-se destacar a ocorrência de um soro com título 64 nesta prova e resultado negativo na combinação SAL+2-ME. Das 61 amostras com resultado inconclusivo na combinação, 55 (90,2\%) apresentaram resultado negativo na RFC e 6 (9,8\%) apresentaram resultado positivo, sendo um deles com título 64 e um com título igual ou superior a 128 . Excluindo as amostras com resultado inconclusivo, verifica-se que, das 490 amostras positivas na RFC, $483(98,6 \%)$ foram também positivas na combinação SAL+2-MEe 7 (1,4\%) foram negativas. Das 510 amostras negativas na RFC, $448(87,8 \%)$ foram negativas também na combinação e $62(12,2 \%)$ foram positivas. A proporção de resultados concordantes entre esses dois recursos de diagnóstico confirmatório foi de $93,1 \%$, resultando em kappa igual a 0,86 , ou seja, concordância ótima.

Tabela 3 - Títulos obtidos na reação de fixação de complemento (RFC) comparados com a combinação dos testes de soroaglutinação lenta (SAL) e 2-mercaptoetanol (2-ME) para diagnóstico sorológico de brucelose em bovinos.

\begin{tabular}{lcccc}
\hline RFC & \multicolumn{3}{c}{ SAL + 2-ME } & Total \\
\cline { 2 - 4 } & Positivo & Inconclusivo & Negativo & \\
\hline Positivo & 483 & 6 & 7 & 496 \\
Negativo & 62 & 55 & 448 & 565 \\
\hline Total & 545 & 61 & 455 & 1.061 \\
\hline
\end{tabular}


Com basena combinação dos resultados daSAL+2ME e da RFC, a sensibilidade relativa do AAT foi $99,6 \%$ e a especificidade relativa foi $83,9 \%$. A RFC apresentou sensibilidade relativa de 91,1\% e especificidade relativa de $100 \%$, quando seus resultados foram confrontados com a condição verdadeira determinada pela associação dos resultados do AAT e da combinaçãoSAL+2-ME. A sensibilidade relativa da combinaçãoSAL+2-MEfoi $98,8 \%$ ea especificidade relativa foi $96,2 \%$, com base na condição verdadeira determinada pela associação do AAT e da RFC.

\section{DISCUSSÃO}

Os testes sorológicos constituem a base do diagnóstico em um programa sanitário para controle ou erradicação da brucelose animal, por proporcionarem uma alternativa que viabiliza o teste de um grande número de amostras. No entanto, deve ser considerado que o diagnóstico sorológico está sujeito a erros, tanto pela ocorrência de resultados falso-positivos quanto pela ocorrência de resultados falso-negativos. $\mathrm{O}$ recurso adotado pelo PNCEBT, para o diagnóstico da brucelose em bovinos e bubalinos, foi a utilização de testes sorológicos em série, usando o teste do AAT na triagem, sendo a confirmação dos resultados positivos realizada pela combinação dos testes SAL+2-ME ou então pela RFC (BRASIL, 2001; 2004). Esse procedimento tem o propósito de melhorar a especificidade do diagnóstico, de modo a minimizar a ocorrência de resultados falso-positivos, o que acarretaria o sacrifício desnecessário de um grande número de animais.

A comparação, no presente estudo, entre os testes adotados pelo PNCEBT mostrou uma concordância boa entre o teste de triagem e os testes confirmatórios. Essa comparação apontou um coeficiente kappa de 0,73 e 0,80 , quando o AAT foi comparado, respectivamente, com a RFC e a combinação SAL+2-ME. Esta, como se pode observar, apresentou a melhor concordância com o teste de triagem. Concordâncias mais elevadas que esta entre o AAT e o 2-ME foram observadas em bovinos porMEgidet al. (2000), kappa =0,84, e porKurodaet al. (2004), kappa =0,94, eem bubalinos, por PinTo et al. (2005), kappa =0,92.

Os valores de kappa entre AAT e RFC encontrados por Dajeretal. (1999) em bovinos ePAulin (2006) em bubalinos, respectivamente, 0,70 e 0,69, foram inferiores aos relatados no presente trabalho (kappa =0,73); já os valores citados por MolNÁr et al. (2002) e Pinto et al. (2005), ambos em bubalinos, foram superiores, respectivamente 0,84 e 0,82. GuARINO etal. (2001), comparando o teste rosa de Bengala, similar ao AAT, com a RFC, obtiveram $88 \%$ de resultados concordantes, e KURODA et al. (2004) observaram
$97,3 \%$, ao passo que no presente trabalho essa proporção foi de $85,7 \%$.

Entretanto, devem-se destacar algumas ocorrências de resultado positivo no teste confirmatório em soros com resultado negativo no teste de triagem (Tabelas 1 e 2), o que mostra a possibilidade de resultado falso-negativo no procedimento adotado pelo PNCEBT. Resultados negativos no AAT e positivos em provas confirmatórias também já foram relatados em outros trabalhos (MATHIAS; PINTO, 1983;SUTHERLAND, 1984; BerCOVICH, 1998; GUARINO et al., 2001), mas essa não é a situação desejada, pois um teste de triagem deve primar pela elevada sensibilidade, pois a ocorrência de resultados falso-negativos pode dificultar a erradicação da enfermidade. Para contornar esse problema, o PNCEBT prevê a repetição periódica dos testes até o completo saneamento do rebanho (BRASIL, 2004). A ocorrência de resultados falso-negativos também foi relatada por NicoletTi (1967), embora o mesmo autor, em outra investigação (NicOLETTI,1992), tenha constatado que o AAT foi capaz de identificar todos os búfalos infectados de um rebanho, eSHANDU; Joshi (1993) e FosGATE et al. (2002) recomendem o AAT como teste de triagem para o controle da brucelose em bovinos e bubalinos, devido à maior sensibilidade, em comparação com a de outros testes.

SHANDU; Joshi (1993) apontaram o 2-ME como um excelente teste confirmatório para o diagnóstico da brucelose. Por outro lado, Nielsen (2002) afirmou que essa prova pode proporcionar resultados falso-negativos em decorrência da presença de pontes dissulfeto também nas moléculas de IgG. OPNCEBT optou pela execução do teste do 2-MEjuntamente com a prova de SAL porque, como aquele teste não detecta $\operatorname{IgM}$, não é adequado para a identificação de infecções recentes, podendo proporcionar resultado negativo em soros com títulos elevados de IgM, os quais podem ser detectados pelo teste de SAL; nesse caso, o resultado é classificado como inconclusivo.

Comparando os resultados dos testes confirmatórios entre si, observou-se concordância ótima quando a RFC foi comparada com a combinaçãoSAL+2-ME (Tabela 3), sendo o kappa igual a 0,86. Na comparação entre RFC e SAL+2-ME, a proporção de resultados concordantes foi de $93,1 \%$, resultados próximos aos encontrados por PAULIN et al. (2002), em bovinos (kappa $=0,88$; proporção de resultados concordantes $=94,6 \%$, e por PINTO et al. (2005), em bubalinos (kappa $=0,86$; proporção de resultados concordantes $=93,3 \%$ ), e inferiores aos obtidos por KuRODA et al. (2004), em bovinos (kappa =0,96; proporção de resultados concordantes $=97,8 \%$ ).

Como esperado, os testes confirmatórios apresentaram melhor concordância entre si do que com o teste de triagem, uma vez que muitos soros com resultado positivo na triagem apresentam resultado negativo 
no teste confirmatório, como consequência da maior especificidade deste. No entanto, não se pode desconsiderar a ocorrência de alguns resultados divergentes entre os testes confirmatórios, com soros apresentando resultado negativo em um teste e título elevado no outro, indicando a possibilidade de ocorrência de falso-negativo nos testes confirmatórios. Esses resultados discordam da constatação feita por Mylrea; Fraser (1976), que observaram correspondência entre os resultados do 2-ME e da RFC a partir da diluição 1:16 neste último teste. NiCOLETTI(1969) e MARIÑoet al. (1991) não observaram diferença significativa entreRFCe2-MEpara detectarvacasinfectadas, mas CoRRÊA DE SÁ (1989) relatou que a RFC não deve ser substituída pelo 2-ME, como teste confirmatório no diagnóstico da brucelose bovina, por não ter sido observada concordância adequada entre os resultados dos dois testes. A ocorrência de resultado negativo na RFC em soro com título alto no 2-ME, observada no presente estudo, poderia estar associada à presença de IgG2, a qual aglutina, mas não fixa complemento (PAulin et al., 2002).

O desempenho do AAT, com sensibilidade relativa de $99,6 \%$ e especificidade relativa de $83,9 \%$, está de acordo com o que se espera de um teste de triagem, ou seja, maior sensibilidade e menor especificidade. Também o desempenho da reação de fixação de complemento, com sensibilidade relativa de 91,1\% e especificidade relativa de $100,0 \%$, é compatível com as características de um teste confirmatório, que deve primar pela maior especificidade. Já os resultados observados para a combinação SAL+2-ME mostraram maior sensibilidade relativa do que especificidade, oqueé diferente daquilo que se espera deum teste confirmatório. Obviamentehá que selevar em conta que estimativa relativa da sensibilidade e da especificidade está mais sujeita a distorções, pela dificuldade dos testes determinarem a condição verdadeira de alguns animais e pelo descarte de amostras resultantes da discrepância entre os testes usados como parâmetro.

\section{CONCLUSÃO}

Os resultados obtidos no presente trabalho mostraram que o teste do antígeno acidificado tamponado apresentou elevada sensibilidade relativa, como se espera de um teste de triagem, e que os dois testes confirmatórios apresentaram elevada especificidade. Os dados obtidos também reforçam a idéia de que o diagnóstico sorológico da brucelose é mais confiável quando obtido por meio dos resultados de mais de um teste, uma vez que soro com título elevado em um teste pode apresentar resultado negativo em outro.

\section{REFERENCIAS}

\section{ABIMERHI, D.; GUTIÉRREZ, A.F.; VILLALOBOS,}

E.J. Uso de las pruebas de ensayo

inmunoabsorbente ligado a enzimas y aglutinación con rivanol para el diagnóstico de brucelosis bovina en Yucatán, México. Veterinária México, v.29, n.2, p.167-171, 1998.

ALTON, G.G.; MAW, J.; ROGERSON, B.A.; MCPHERSON, G.G. The serological diagnosis of bovine brucellosis: an evaluation of the complement fixation test, serum agglutination and rose Bengal test. Australian Veterinary Journal, v.51, n.2, p.57-63, 1975.

ALTON, G.G.; JONES, L.M.; ANGUS, R.D.; VERGER, J.M. Techniques for the brucellosis laboratory. Paris: Institut Nacional de la Recherche Agronomique, 1988. 190p.

BERCOVICH, Z. Maintence of Brucella abortus-free herds: a review with emphasis on the epidemiology and the problems in diagnosing brucellosis in areas of low prevalence. Veterinary Quarterly, v.20, n.3, p.81-88, 1998.

BRASIL, Ministério da Agricultura e do Abastecimento. Departamento de Defesa Animal. Instrução Normativa no 2, de 10 de janeiro de 2001. Institui o Programa Nacional de Controle e Erradicação da Brucelose e Tuberculose (PNCEBT). Republicada no Diário Oficial da República Federativa do Brasil, Poder Executivo, 16 jan. 2001. Secção 1, p. 11-17.

BRASIL, Ministério da Agricultura, Pecuária e Abastecimento. Instrução normativa SDA n-6, de 08 de janeiro de 2004. Aprova o Regulamento Técnico do Programa Nacional de Controle e Erradicação da Brucelose e Tuberculose Animal. Diário Oficial da República Federativa do Brasil, Poder Executivo, Brasília, DF, 12 de janeiro de 2004. Seção 1, p.6-10.

BRASIL, Ministério da Agricultura, Pecuária e Abastecimento. Departamento de Saúde Animal, 2006. Programa Nacional de Controle e Erradicação da Brucelose e Tuberculose (PNCEBT) - Manual Técnico. Brasília: MAPA / DAS / DSA, 2006, 188p.

CORNER, L.A.; ALTON, G.G.; MCNICHOL, L.N.; STREETENS, T.; TRUEMEN, K.F. An evaluation of an anamnestic test for brucellosis in cattle of the northern pastoral areas. Australian Veterinary Journal, v.60, n.1, p.1-3, 1983.

CORRÊA DE SÁ, M.I.V.C. Brucelose bovina Avaliação da prova de imunodifusão radial com o antígeno hapteno nativo (HN) no diagnóstico sorológico da brucelose em animais infectados e vacinados. Estudo comparativo com outras provas sorológicas. Repositório de Trabalhos do L.N.I.V., v.21, p.11-44, 1989. 
DAJER, A.; LUNA-MARTÍNEZ, E.; ZAPATA, D.; VILLEGAS, S.; GUTIÉRREZ, E.; PEÑA, G.; GURRÍA, F.; NIELSEN, K.; GALL, D. Evaluation of a fluorescencepolarization assay for the diagnosis of bovine brucellosis en México. Preventive Veterinary Medicine, v.40, n.1, p.67-73, 1999.

FOSGATE, G.T.; ADESIYUN, A.A.; HIRD, D.W.; JOHNSON, W.O.; HIETALA, S.K.; SCHURIG, G.G.; RYAN, J. Comparison of serologic test for detection of Brucella infections in cattle and water buffalo (Bubalus bubalis). American Journal of Veterinary Research, v.63, n.11, p.1598-1605, 2002.

GUARINO, A.; FUSCO, G.; DI MATTEO, A.; URBANI, G.; CONDOLEO, R.; SERPE, L.; TITTARELLI, M.; DI VENTURA, M.; GALLO, P. Indirect ELISA for the diagnosis of brucellosis in water buffaloes (Bubalus bubalis) in Italy. Veterinary Record, v.149, p.88-90, 2001.

HAYES, J.; CHAPPEL, R.J. A comparison of the results of the brucellosis radioimmunoassay and other serological test in experimentally infected cattle. Journal of Hygiene, v.88, n.1, p.21-28, 1982.

HUBER, J.D.; NICOLETTI, P. Comparison of the results of card, rivanol, complement-fixation, and milk ring tests with the isolation rate of Brucella abortus from cattle. American Journal of Veterinary Research, v.47, n.7, p.1529-1531, 1986

KURODA, R.B.S.; PAULIN, L.M.S.; NOZAKI, C.N.; SILVA JUNIOR, F.F.; GERONUTTI, MEGID, J. Prevalência da brucelose bovina na microrregião da serra de Botucatu - Estudo comparativo dos resultados das técnicas de soroaglutinação lenta em tubos, 2mercaptoetanol e fixação de complemento. Arquivos do Instituto Biológico, São Paulo, v.71, n.2, p.137-142, 2004.

MARIÑO, J.; GALLEGO, M.M.I.; DELEÓN, L.S.; ALMANSA, M.J. Comparación de técnicas serológicas en la evaluación de bovinos infectados naturalmente por Brucella abortus. In: FRANK, J.F. (Ed.). Networking in brucellosis research. Tokyo: United Nations University Press, 1991. p.120-30. (Report of the United Nations University, Brucellosis Research Network).

MARTIN, S.W.; MEEK, A.H.; WILLEBERG, P. Veterinary epidemiology. Ames: Iowa State University Press, 1987. $343 p$.

MATHIAS, L.A.; PINTO, A.A. Serological diagnosis of brucelosis in water buffaloes (Bubalu bubalis): comparison among complement fixation, serum agglutination and rose Bengal test. International Journal os Zoonosis, v.10, p.122-126, 1983.

MATHIAS, L.A.; MacMILLAN, A.P.; GREISER-WILKE, I.; MOENNING, E.V. Comparação entre a reação de fixação de complemento, teste imunoenzimático indireto e teste imunoenzimático competitivo no diagnóstico sorológico da brucelose em bovinos procedentes de rebanhos com histórico da enfermidade. Ars Veterinária, v.11, n.1, p.47-55, 1995.

MEGID, J.; RIBEIRO, M.G.; MARCOS JÚNIOR, G.; CROCCI, A.J. Evaluation of rapid agglutination, tube agglutination, buffered plate antigen and 2mercaptoethanol tests in the diagnosis of bovine brucellosis. Brazilian Journal of Veterinary Research and Animal Science, v.37, n.4, p.395-399, 2000.

MOLNÁR, L.; MOLNÁR, E.; LIMA, E.S.C.; DIAS, H.L.T. Avaliação de seis testes sorológicos no diagnóstico da brucelose bubalina. Pesquisa Veterinária Brasileira, v.22, n.2, p.41-44, 2002

MYLREA, P.J.; FRASER, G.C. The use of supplementary tests in the serological diagnosis of bovine brucellosis. Australian Veterinary Journal, v. 52, p. 261-266, 1976.

NICOLETTI, P. Utilization of the card test in brucellosis eradication. Journal of the American Veterinary Medical Association, v.151, n.12, p.1778-1783, 1967.

NICOLETTI, P. Further evaluations of serologic test procedure used to diagnose brucellosis. American Journal of Veterinary Research, v.30, n.10, p.1811- 1816, 1969.

NICOLETTI, P. An evaluation of serologic tests used to diagnose brucellosis in buffaloes (Bubalus bubalis). Tropical Animal Health Production, v.24, n.1, p.40-44, 1992.

NICOLETTI, P.; TANYA, V. Comparison of enzymelabeled immunosorbent assay and particle concentration fluorescence immunoassay with standard serologic methods and bacteriologic culture for detection of Brucella sp.-infected cows in herds with brucellosis. Journal of the American Veterinary Medical Association, v.202, n.12, p.1975-1977, 1993

NIELSEN, K. Diagnosis of brucellosis by sorology. Veterinary Microbiology, v.90, p.447-459, 2002.

PAULIN, L.M.; PRADO, G.E.S.; FEDERSONI, I.S.P.; TEIXEIRA, A.C.; CASTRO, V.; GENOVEZ, M.E. Estudo comparativo dos testes 2-mercaptoetanol e reação de fixação do complemento no sorodiagnóstico da brucelose bovina. Arquivos do Instituto Biológico, São Paulo, v.69, n.4, p.41-47, 2002.

PAULIN, L.M.S. Estudo comparativo de diferentes técnicas sorológicas para diagnóstico de infecções por Brucella abortus em búfalos (Bubalus bubalis). 2006. 92p. Tese (Doutorado) - Faculdade de Medicina Veterinária e Zootecnia da Universidade de São Paulo, São Paulo, 2006.

PEREIRA, M.G. Epidemiologia. Teoria e prática. Rio de Janeiro: Guanabara Koogan, 2000. 596p. 
PINTO, M.R.A.; FAGLIARI, J.J.; MATHIAS, L.A.; MEGID, J.; SALGADO, V.R. Avaliação da prova do antígeno acidificado tamponado, em comparação com as provas de fixação de complemento e 2mercaptoetanol, para diagnóstico sorológico da brucelose em um rebanho bubalino (Bubalus bubalis) infectado por Brucella abortus. Ars Veterinaria, v.21, p.147-154, 2005. Suplemento.

SHANDU, K.S.; JOSHI, D.V. Comparative study in cattle and buffaloes for evaluation of various diagnostic tests for brucellosis. Indian Journal of Pathology and Microbiology, v.36, n.4, p.458-465, 1993.
SUTHERLAND, S.S. Evaluation of the enzyme-linked immunosorbent assay in the detection of cattle infected with Brucella abortus. Veterinary Microbiology, v.10, p.23-32, 1984.

SUTHERLAND, S.S; LE GRAS, D.V.; ROBERTSON, A.G.; JOHNSTON, J.M.; EVANS, R.J. Serological response of cattle after vaccination and challenge with Brucella abortus. Veterinary Microbiology, v.7, n.2, p.165-175, 1982.

Recebido em $12 / 9 / 08$

Aceito em 18/3/10 